\title{
HIDROCARBONETOS POLICÍCLICOS AROMÁTICOS ATMOSFÉRICOS DE FONTES AUTOMOTIVAS: UMA BREVE REVISÃO
}

\author{
J. A. H. Azevedo', R. S. Araújo ${ }^{2}$, G. M. M. Silva ${ }^{3}$ \\ $1,2,3$ Instituto Federal do Ceará \\ julieholandaazevedo@gmail.com ${ }^{1}$ - rinaldo@ifce.edu.br ${ }^{2}$ - gloriamarinho@ifce.edu.br ${ }^{3}$
}

Artigo submetido em janeiro/2013 e aceito em março/2013

\section{RESUMO}

A ocorrência de hidrocarbonetos policíclicos aromáticos (HPAs) no ambiente tem se tornado preocupante, por eles serem abundantes e possíveis carcinógenos humanos. HPAs e seus derivados são produzidos pela combustão incompleta da matéria orgânica, sendo a queima de combustíveis fósseis uma das principais fontes desses poluentes no meio ambiente. As concentrações de HPAs aumentam significativamente em ambientes urbanos e são influenciadas principalmente pelas emissões veiculares. Os HPAs em fase gasosa são encontrados a baixos níveis no ar atmosférico, apresentando-se comumente associado às partículas finas em suspensão. Esta revisão descreve os HPAs, provenientes de emissões veiculares, quanto as suas características de amostragem e análise, identificação, legislação e saúde ambiental. Oportunamente, alguns países propõem limites de concentração não obrigatórios para os HPAs, porém muitos estudos relacionados à exposição a estes compostos mostram que estes poluentes devem ser prioritários quando se considera a qualidade do ar e o risco à saúde humana.

PALAVRAS-CHAVE: HPAs, fontes móveis, material particulado, amostragem, legislação e saúde.

\section{POLYCYCLIC AROMATIC HYDROCARBONS ATMOSPHERIC FROM AUTOMOTIVE SOURCES: A BRIEF REVIEW}

\begin{abstract}
The occurrence of polycyclic aromatic hydrocarbons (PAHs) in the environment has become alarming because they are abundant and possible human carcinogens. PAHs and derivatives are produced by incomplete combustion of organic material, and the burning of fossil fuels is one of the main sources of these pollutants into the environment. The Concentrations of PAHs increased significantly in urban environments and are influenced mainly by vehicle emissions. PAHs are found in the gas phase at low levels, however, the most
\end{abstract}

likely human carcinogen is found associated with particles, especially fine particulate matter in ambient air. This review characterizes the PAHs from vehicle emissions, with a focus on aspects of sampling and analysis, identification, health and legislation related to these compounds. Some countries propose concentration limits required for $\mathrm{PAH}$, but many studies regarding exposure PAHs show that these pollutants should be a priority when considering air quality and human health risk.

KEY-WORDS: PAHs, mobile sources, particulate matter, sampling, legislation and health. 
HIDROCARBONETOS POLICÍCLICOS AROMÁTICOS ATMOSFÉRICOS DE FONTES AUTOMOTIVAS: UMA BREVE REVISÃO

\section{INTRODUÇÃO}

Hidrocarbonetos policíclicos aromáticos (HPAs) são uma classe de compostos orgânicos contendo dois ou mais anéis benzênicos condensados, podendo também apresentar anéis com menos de seis carbonos ${ }^{1}$. São caracterizados pela baixa solubilidade em água e alto coeficiente de partição octanol/água e elevada hidrofobicidade natural ${ }^{2}$.

Os HPAs são considerados os contaminantes orgânicos mais comuns no meio ambiente e foram os primeiros a serem observado quanto aos seus efeitos biológicos em termos de potencial carcinogênico, mutagênico, teratogênico e demais efeitos tóxicos que possam afetar a saúde em geral ${ }^{3,4}$.

A Agência de Proteção Ambiental dos Estados Unidos (US EPA) ${ }^{5}$ classificou em 1994 os HPAs como poluentes B-2, que significa provável carcinogênico humano, sendo o benzo[a]pireno a espécie indicadora. Com o aumento do peso molecular desses compostos a carcinogenicidade aumenta e a toxicidade aguda diminui ${ }^{6}$. Os HPAs prioritários estão listados na Tabela 01 a seguir.

Tabela 1: HPAs prioritários segundo a US EPA e algumas propriedades físico-químicas.

\begin{tabular}{|c|c|c|c|c|c|}
\hline COMPOSTOS & $\begin{array}{c}\text { NÚMERO } \\
\text { DE ANÉIS }\end{array}$ & $\begin{array}{c}\text { MASSA } \\
\text { MOLECULAR } \\
(\mathrm{g} / \mathrm{mol})\end{array}$ & $\begin{array}{c}\text { SOLUBILIDADE } \\
(\mathrm{mg} / \mathrm{L})\end{array}$ & $\begin{array}{c}\text { PRESSÃO } \\
\text { DE VAPOR } \\
(\mathrm{mmHg})\end{array}$ & $\begin{array}{c}\text { ESTRUTURA } \\
\text { MOLECULAR }\end{array}$ \\
\hline NAFTALENO & $\mathbf{2}$ & $\mathbf{1 2 8 , 1 7}$ & $\mathbf{3 1}$ & $\mathbf{8 , 8 9 \times 1 0 ^ { - 2 }}$ \\
\hline ACENAFTENO & $\mathbf{3}$ & $\mathbf{1 5 4 , 2 1}$ & $\mathbf{3 , 8}$ & $\mathbf{3 , 7 5 \times 1 0 ^ { - 3 }}$ \\
\hline ACENAFTALENO & $\mathbf{3}$ & $\mathbf{1 5 2 , 2 0}$ & $\mathbf{1 6 , 1}$ & $\mathbf{2 , 9 0 \times 1 0 ^ { - 2 }}$ \\
\hline ANTRACENO & $\mathbf{3}$ & $\mathbf{1 7 8 , 2 3}$ & $\mathbf{0 , 0 4 5}$ & $\mathbf{2 , 5 5 \times 1 0 ^ { - 5 }}$ \\
\hline FENANTRENO & $\mathbf{3}$ & $\mathbf{1 7 8 , 2 3}$ & $\mathbf{1 , 1}$ & $\mathbf{6 , 8 0 \times 1 0 ^ { - 4 }}$ \\
\hline FLUORENO & $\mathbf{3}$ & $\mathbf{1 6 6 , 2 2}$ & $\mathbf{1 , 9}$ & $\mathbf{3 , 2 4 \times 1 0 ^ { - 3 }}$ \\
\hline & & & $\mathbf{2 0 2 , 2 6}$ & $\mathbf{0 , 2 6}$ & $\mathbf{8 , 1 3 \times 1 0 ^ { - 6 }}$ \\
\hline & & & & \\
\hline
\end{tabular}




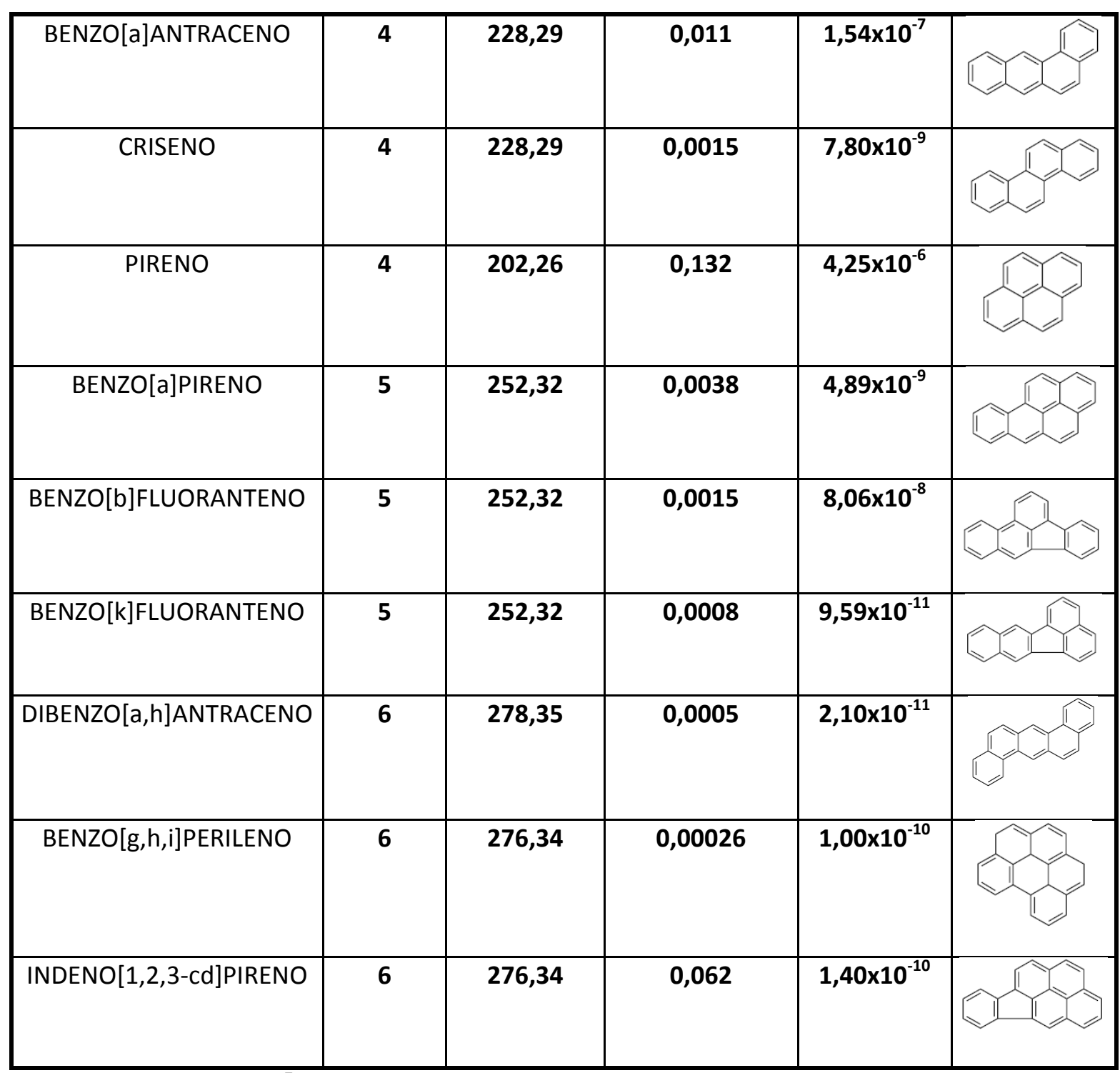

Fonte: Adaptado de Bojes ${ }^{7}$ et al. (2007).

Os HPAs podem ser originados de fontes domésticas, móveis, industriais, agrícolas e naturais $^{6}$ e são formados a partir da combustão incompleta e pirólise da matéria orgânica, sendo a queima de combustíveis fósseis uma das principais fontes antropogênicas de HPAs na atmosfera ${ }^{8}$. HPAs de baixo peso molecular, com dois ou três anéis aromáticos, são emitidos na fase gasosa, enquanto que, os de peso molecular elevado, com cinco ou mais anéis, são emitidos na fase particulada, associados às partículas em suspensão ${ }^{6,9}$.

As fontes domésticas são caracterizadas pela queima de madeira usada para aquecimento $^{10}$, sendo a contribuição de cerca de $30 \%$ no inverno e praticamente inexistente no verão $^{11}$. A poluição do ar em ambientes fechados nos países em desenvolvimento é um grande desafio à saúde pública e ambiental, cerca de $50 \%$ das pessoas dependem do carvão e da biomassa na forma de esterco, madeira e resíduos da lavoura para produção de energia doméstica ${ }^{12}$. A exposição à poluição do ar nesses ambientes pode ser responsável por quase dois milhões de mortes em países em desenvolvimento e cerca de $4 \%$ da carga global de doenças ${ }^{13,14}$. Em áreas rurais, compostos como hidrocarbonetos, tióis, aldeídos, cetonas e HPAs representam cerca de $30 \%$ a $50 \%$ da massa de material particulado de $10 \mu \mathrm{m}$ (MP 10$)^{15,16}$. O fenantreno é o composto mais abundante e frequentemente detectado em amostras domésticas ${ }^{17}$. 
As fontes industriais comprometem intensamente a qualidade do ar e isso é cada vez mais frequente em países industrializados ${ }^{18}$. A falta de controle das emissões industriais e o aumento de veículos mal conservados determina que a maioria dos países em desenvolvimento sofra de grave poluição do ar exterior ${ }^{19}$.

As fontes naturais de HPAs incluem o carvão, o petróleo bruto, vulcões e incêndios ${ }^{20}$. HPAs não são tóxicos apenas nas suas formas nativas, mas também podem produzir produtos cancerígenos após reações com outras espécies atmosféricas, como, por exemplo, quando reagem com o óxido de nitrogênio formando nitro-HPAs ${ }^{21}$.

As fontes móveis incluem as emissões veiculares, como aeronaves ${ }^{22}$, navegação ${ }^{23,24}$, ferrovias ${ }^{25}$ e automóveis ${ }^{26}$. O tipo e a idade do motor, de combustível e a partida a frio são alguns fatores que influenciam na de emissão de HPAs ${ }^{27}$.

Neste contexto devido a grande ocorrência atmosférica de HPAs, principalmente decorrente do uso crescente de combustíveis fósseis e os riscos atribuídos à saúde, comuns a esta classe de compostos químicos, o presente estudo revisa a literatura existente relacionada aos HPAs provenientes de emissões veiculares e suas relações com o meio ambiente.

\section{HPAs EM FONTES AUTOMOTIVAS}

A elevada demanda de combustíveis fósseis, como consequência do aumento da população, da crescente taxa de urbanização e do tráfego de veículos, durante as últimas décadas $^{28}$ têm causado grandes emissões de HPAs na atmosfera ${ }^{29}$, gerando grandes impactos à saúde humana, como cânceres e mutações genéticas ${ }^{1}$.

A produção de HPAs por fontes automotivas à gasolina depende da relação ar/combustível e a quantidade de HPAs no escape do motor diminui quanto mais pobre for a mistura ${ }^{30,31}$. Em geral, o perfil de concentração e a proporção de HPAs podem ser utilizados para determinar a contribuição das diferentes fontes ${ }^{32}$. Níveis maiores de benzo[ghi]pireno, coroneno e fenantreno foram propostos para as emissões de veiculares ${ }^{33}$. Miguel $^{34}$ et al. (1998) constataram que os caminhões a diesel são as principais fontes de HPAs mais leves e veículos a gasolina são as principais fontes de HPAs de maior peso molecular, tais como benzo[a]pireno $(B[a] P)$ e dibenzo[a,h]antraceno. Marr ${ }^{27}$ et al. (1999) encontraram contribuições significativas na atmosfera para HPAs de 4 e 5 anéis benzênicos provenientes de motores a gasolina, enquanto para motores a diesel foram detectados HPAs de 2 e 3 anéis benzênicos, como o naftaleno e o fluoranteno.

A combustão de óleo foi relatada e associada a concentrações elevadas de HPAs mais voláteis $\left(120 \mathrm{ng} / \mathrm{m}^{3}\right)$, como fluoreno, fluoranteno e pireno, em conjunto com níveis moderados de compostos de maior peso molecular $\left(19 \mathrm{ng} / \mathrm{m}^{3}\right)$, como benzo[b]fluoranteno e indeno[1,2,3cd]pireno ${ }^{35}$.

Segundo Bourotte ${ }^{36}$ et al. (2005), o método da razão binária foi usado na identificação das fontes de HPAs e envolve relações de pares de compostos frequentemente encontrados em emissões de HPAs. Estes autores encontraram razões Benzo[a]Pireno/Benzo[ghi]Pireno de 0,25, Indeno/Benzo[ghi]Pireno de 0,97 e Benzo[ghi]Pireno/Indeno de 0,94 em emissões de HPAs provenientes de emissões veiculares, os quais são similares aos relatados por Simcik ${ }^{37}$ et al. (1999) e De Martins ${ }^{38}$ et al. (2002). Os autores ainda sugerem que razões entre Indeno/Benzo[ghi]Pireno $(0,97)$ e Benzo[a]Antraceno/Benzo[a]Pireno $(0,85)$ se relacionam a uma 
contribuição da queima de diesel, assim como as razões entre Benzo[e]Pireno/Benzo[a]Pireno $(1,11)$ e Fluoreno/(Fluoreno+Pireno) $(0,52)$ estão associadas à queima de gasolina. Em termos, estes valores corroboram a teoria de Mainwaring e Stirling ${ }^{39}$ (1981), que a proporção de HPAs pode ser utilizada para distinguir os perfis de tráfego dentre as diversas fontes emissoras.

Azimi $^{40}$ et al. (2005) mostraram que os níveis totais de HPAs em áreas urbanas da França foram semelhantes (153 a $161 \mu \mathrm{g} \mathrm{m}^{-2} \mathrm{y}^{-1}$ ) a níveis encontrados em uma região urbana no norte da Grécia (73-401 $\mu \mathrm{g} \mathrm{m}^{-2} \mathrm{y}^{-1}$ ) por Manoli ${ }^{41}$ et al. (2002). Park ${ }^{42}$ et al. (2001) e Lang $^{43}$ et al. (2002) encontraram 363 e $230 \mu \mathrm{g} \mathrm{m}^{-2} \mathrm{y}^{-1}$ em Miami e no Texas, respectivamente, mostrando que os fluxos de deposição total de HPAs são semelhantes em várias áreas urbanas do hemisfério norte. Fenantreno, fluoranteno e pireno foram identificados por Azimi ${ }^{40}$ et al. (2005) como os compostos mais abundantes, confirmando a origem pirolítica de compostos aromáticos na deposição atmosférica ${ }^{44,45}$. O impacto da contaminação pirolítica também é sugerido pela presença de criseno e benzo[a]antraceno, que são considerados como marcadores de processos de combustão ${ }^{46}$.

HPAs associados ao material particulado de $10 \mu \mathrm{m}$ (MP10) na cidade de São Paulo, Brasil, foram relatados por De Martinis ${ }^{38}$ et al. (2002) em 1994 e por Vasconcellos ${ }^{47}$ et al. (2003) em 2000 , sendo encontrado por estes autores valores da ordem de $95,5 \mathrm{ng} / \mathrm{m}^{3}$ e $122 \mathrm{\mu g} / \mathrm{m}^{3}$, respectivamente. Estes valores confirmaram o aumento considerável de material particulado fino na atmosfera da capital paulista no período. Segundo Bourotte et al. $(2005)^{36}$, o aumento dos níveis de HPAs na atmosfera nos grandes centros urbanos está associado à intensa urbanização e ao aumento do tráfego de veículos automotores.

No Rio de Janeiro, Fernandes ${ }^{48}$ et al. (2002) encontraram as maiores concentrações de HPAs na Avenida Brasil $\left(11,5 \mathrm{ng} / \mathrm{m}^{3}\right)$, no período de dezembro de 1998 a março de 1999 . Os autores sugerem que os níveis de HPAs relativamente baixos são provavelmente consequência das condições meteorológicas do período, que aumentaram os mecanismos de deposição/dispersão e remoção química.

Baixos níveis de HPAs foram relatados por Teixeira ${ }^{49}$ et al. (2012) no período de 2006 a 2008 em Porto Alegre, no Rio Grande do Sul $\left(2,1 \mathrm{ng} / \mathrm{m}^{3}\right)$. Neste caso, os autores sugeriram que estas baixas concentrações estão relacionadas às condições meteorológicas locais, já que o verão é caracterizado por velocidades mais elevadas do vento, temperaturas mais altas e maior incidência de radiação, o que favorece a dispersão e degradação fotoquímica dos HPAs.

\subsection{AMOSTRAGEM E ANÁLISE}

Níveis reais de HPAs no ar ambiente devem ser revelados através de amostras representativas. $\mathrm{O}$ monitoramento desses compostos geralmente é feito usando amostradores de baixo ${ }^{21}$, médio ${ }^{50}$ ou grande volume ${ }^{51}$, no qual o ar é forçado por meio de bombas, passando por adsorventes e filtros, onde são retidos certos componentes da fase gasosa e as partículas em suspensão ${ }^{52}$.

Na amostragem de fases gasosa e particulada é importante que os adsorvente e filtros apresentem uma elevada eficiência de retenção, grande capacidade de absorção e adsorção e estabilidade química. As espumas de poliuretano, o XAD-2, o XAD-4, o florisil e o seppark-C18 são os adsorventes mais frequentemente utilizados para a retenção de HPAs em fase gasosa ${ }^{53-58}$.

Para a retenção dos HPAs associados ao material particulado são utilizados filtros de fibra 
de vidro, filtros de fibra de quartzo e politetrafluoretileno (PTFE) ${ }^{59-61}$, sendo este o mais comumente utilizado e indicado, pois é quimicamente inerte e estável, além de apresentar grande resistência à ação de solventes orgânicos ${ }^{62}$.

Para a identificação e quantificação dos HPAs são comumente usadas as técnicas analíticas da cromatografia gasosa $(C G)^{63,64}$ e da cromatografia líquida de alta eficiência (CLAE) ${ }^{65}$ acopladas a espectrômetro de massa (MS) e a detectores de fluorescência (FLD), no ultravioleta (UV), com arranjo de diodos (DAD) e por ionização de chama (FID) $)^{52}$.

\subsection{SAÚDE E LEGISLAÇÃO}

Alguns países já acrescentaram os HPAs em suas listas de compostos perigosos, porém não há um rigoroso padrão de qualidade do ar para tais moléculas. $\mathrm{O} B$ [a]P é usado como um marcador de risco potencial a saúde ${ }^{1}$.

As principais fontes de $\mathrm{B}[\mathrm{a}] \mathrm{P}$ no ambiente incluem o transporte rodoviário, o uso de combustíveis sólidos domésticos e as atividades siderúrgicas. A principal fonte de exposição humana é atribuída a fumaça de cigarro. Estudos de exposição ocupacional a HPAs mostraram um aumento da incidência de tumores da pele, pulmão, bexiga e outros órgãos. O câncer de pulmão está frequentemente associado à inalação de $\mathrm{HPAs}^{4,66}$. Os HPAs variam na sua capacidade de induzir tumores em animais ou humanos ${ }^{67-70}$.

A US EPA classificou os HPAs, em 1994, sendo o $\mathrm{B}$ [a]P relacionado como carcinógeno com forte evidência em animais, mas com evidências insuficientes para humanos. A Occupational Safety and Health Administration (OSHA) estabeleceu um limite de $0,2 \mathrm{mg} / \mathrm{m}^{3}$ de HPAs ${ }^{71}$ para segurança dos possíveis efeitos à saúde humana. A Diretiva da União Europeia propôs $1 \mathrm{ng} / \mathrm{m}^{3} \mathrm{de}$ Benzo[a]pireno para a fração total de material particulado de $10 \mu \mathrm{m}$, calculado para um período de um $a n o^{72}$. Países como a Holanda, adota o valor limite de $5 \mathrm{ng} / \mathrm{m}^{3}$ para o marcador $\mathrm{B}[\mathrm{a}] \mathrm{P}^{32}$. Já o Reino Unido recomenda $0,25 \mathrm{ng} / \mathrm{m}^{3}$ para mesmo marcador ${ }^{73}$. No Brasil, não há legislação específica para os HPAs, sendo considerados apenas os hidrocarbonetos totais.

\subsection{OUTRAS TECNOLOGIAS}

A emissão de HPAs por fontes automotivas é uma das principais formas de liberação de HPAs no ar ambiente. Segundo Alkurdi ${ }^{28}$ et al. (2013), o uso de conversores catalíticos em motores à gasolina diminui a emissão automotiva destes poluentes orgânicos, obtendo uma redução de aproximadamente 25 vezes ${ }^{74,75}$. Já para motores a diesel esses conversores catalíticos não são tão eficientes, como mostrado por Rogge ${ }^{76}$ et al. (1993).

Ying $^{77}$ et al. (2006) afirmaram que reformulação do combustível para motores a diesel, envolvendo a redução do teor de enxofre, a diminuição da adição de compostos aromáticos e a adição de oxigênio ao combustível são algumas considerações tecnológicas que podem reduzir as emissões de HPAs na atmosfera.

O uso de compostos oxigenados, como álcoois, ésteres e éteres, se mostra eficaz na redução das emissões de partículas a partir de motores à gasolina. Segundo Abrantes ${ }^{78}$ et al. (2009) o uso do etanol como combustível e/ou a adição deste à gasolina pode reduzir a emissão de HPAs em até $92 \%$. Ainda na classe dos oxigenados, o éter dimetílico é considerado um combustível alternativo promissor, com a vantagem adicional de apresentar um elevado número de cetano ${ }^{79}$. 
$\mathrm{He}^{80}$ et al. (2010) e Surawski ${ }^{81}$ et al. (2011) sugeriram o uso de combustíveis alternativos, como o biodiesel obtido a partir de oleaginosas e/ou sua mistura com o diesel, para a diminuição considerável das emissões de HPAs em motores a diesel.

\section{CONCLUSÕES}

Hidrocarbonetos policíclicos aromáticos e partículas em suspensão tornaram-se abundantes ao longo das décadas devido às ações antropogênicas, sendo importante identificar e monitorar tais contaminantes orgânicos na atmosfera, especialmente os níveis de HPAs. Em geral, o controle e a redução da contaminação por HPAs supõe o conhecimento da natureza, origem, extensão da poluição decorrente e dos riscos à saúde humana que podem ser produzidos, principalmente em centros urbanos populosos onde há elevados níveis de emissão veicular.

\section{AGRADECIMENTOS}

À Fundação Cearense de Apoio ao Desenvolvimento Científico e Tecnológico (FUNCAP) pelo auxílio financeiro concedido.

\section{REFERÊNCIAS BIBLIOGRÁFICAS}

1. Ravindra, K.; Mital, A. K.; Grieken, R. van. Health Risk Assessment of Urban Suspended Particulate Matter with Special Reference to Polycyclic Aromatic Hydrocarbons: A Review. Review on Environmental Health, v.16, p.169-189, 2001.

2. Meador, J. P.; Casillas, E.; Sloan, C. A.; Varanasi, U. Comparative bioaccumulation of polycyclic aromatic hydrocarbons from sediment by two infaunal invertebrates. Marine Ecology Progress Series, v.123, p.107-124, 1995.

3. Agency for Toxic Substances and Disease Registry (ATRSD). Polycyclic aromatic hydrocarbons (PAHs). US Department of Health and Human Services, Public Health Service. Atlanta, 1996.

4. Agency for Toxic Substances and Disease Registry (ATRSD). Support document to the 2011 priority list of hazardous substances that will be the subject of toxicological profiles. Division of Toxicology and Environmental Medicine, Atlanta, 2011.

5. Environmental Protection Agency (EPA). Peer review and peer involvement at the US Environmental Protection Agency, Science Policy Council, Office of the Science Advisor, Environmental Protection Agency, 1994.

6. Ravindra, K.; Sokh, R.; Grieken, R. van. Atmospheric polycyclic aromatic hydrocarbons: Source attribution, emission factors and regulation. Atmospheric Environment, v.42, p.2895-2921, 2008.

7. Bojes, H. K.; Pope, P. G. Characterization of EPA's 16 priority pollutant polycyclic aromatic hydrocarbons (PAHs) in tank bottom solids and associated contaminated soils at oil exploration and production sites in Texas. Regulatory Toxicology and Pharmacology, v.47, p.288-295, 2007. 
8. Tavares Júnior, M.; Pinto, J. P.; Souza, A. L.; Scarmínio, I. S.; Solci, M. C. Emission of polycyclic aromatic hydrocarbons from diesel engine in a bus station, Londrina, Brazil. Atmospheric Environment, v.38, p.5039-5044, 2004.

9. Ramírez, N.; Cuadras, A.; Rovira, E.; Marcé, R. M.; Borrull, F. Risk Assessment Related to Atmospheric Polycyclic Aromatic Hydrocarbons in Gas and Particle Phases near Industrial Sites. Environmental Health Perspectives, v.119, n.8, 2011.

10. Rogge, W. F.; Hildemann, L. M.; Mazurek, M. A.; Cass, G. R. Sources of fine organic aerosol. 9. Pine, oak, and synthetic log combustion in residential fireplaces. Environment Science Technology, v.32, p.13-22, 1998.

11. Pistikopoulos, P.; Masclet, P.; Mouvier, G. A receptor model adapted to reactive species: polycyclic aromatic hydrocarbons; evaluation of source contributions in an open urban site-I. Particle compounds. Atmospheric Environment, v. 24A, n. 5, p.1189-1197, 1990.

12. Jenkins, B. M.; Jones, A. D.; Turn, S. Q.; Williams, R. B. Particle concentrations, gas-particle partitioning, and species intercorrelations for polycyclic aromatic hydrocarbons (PAH) emitted during biomass burning. Atmospheric Environment, v.30, n.22, p.3825-3835, 1996.

13. Rajput, N.; Lakhani, A. Measurements of polycyclic aromatic hydrocarbons in an urban atmosphere of Agra, India. Atmósfera, v.23, n.2, p.165-183, 2010.

14. Callén, M. S.; Cruz, M. T. de la; López, J. M.; Mastral, A. M. PAH in airborne particulate matter. Carcinogenic character of PM10 samples and assessment of the energy generation impact. Fuel Processing Technology, v.92, p.176-182, 2011.

15. Chow, J.C.; Waston, J. G.; Lowenthal, D. H.; Soloman, P. A.; Magliano, K. L.; Ziman, S. D.; Richards, L. W. PM10 and PM2.5 composition in California Sari Joaquin valley. Aersal Science and Technology, v.18, p.105-128, 1993.

16. Chow, J. C.; Watson, J. G.; Fujita, E. M.; Lu, Z. Q.; Oawson, D. R.; Ashbaugh, L. L. Temporal and spatial variation of PM2.5 and PM10 aerosol in the southern California air quality study. Atmospheric Environment, v.28, p.2061-2080, 1994.

17. Shane, B. S.; Henry, C. B.; Hotchkiss, J. H.; Klausner, K. A.; Gutenmann, W. H; Lisk, D. J. Organic toxicants and mutagens in ashes from eighteen municipal refuse incinerators. Arch Environ Contam Toxicol, v.19, n.5, p.665-673, 1990.

18. Klockow, D.; Targa, H. J. Performance and results of a six-year German/Brazilian research Project in the industrial área of Cubatão/SP, Brazil. Pure and Applied Chemistry, v.70, p.22872293, 1998.

19. Van Metre, P. C.; Mahler, B. J.; Furlong, E. T. Urban sprawl leaves its PAH signature. Environmental Science and Technology, v.34, p.4064-4070, 2000.

20. Baek, S. O.; Field, R. A.; Goldstone, M. E.; Kirk, P. W.; Lester, J. N.; Perry, R. A review of atmospheric polycyclic aromatic hydrocarbons: sources, fate and behavior. Water, Air and Soil Pollution, v.60, p.279-300, 1991.

21. Allen, A. G.; Rocha, G. O. da.; Cardoso, A. A.; Machado, C. M. D.; Andrade, J. B. de. Atmospheric particulate polycyclic aromatic hydrocarbons from road transport in southeast Brazil. Transportation Research, Part D, v.13, p.483-490, 2008. 
22. Chen, Y. C.; Lee, W. J.; Uang, S. N.; Lee, S. H.; Tsai, P. J. Characteristics of polycyclic aromatic hydrocarbon (PAH) emissions from a UH-1H helicopter engine and its impact on the ambient environment. Atmospheric Environment, v.40, p.7589-7597, 2006.

23. Cooper, D. A. Exhaust emissions from high speed passenger ferries. Atmospheric Environment, v.35, p.4189-4200, 2001.

24. Cooper, D.A. Exhaust emissions from ships at berth. Atmospheric Environment, v.37, p.38173830, 2003.

25. Devos, O.; Combet, E.; Tassel, P.; Paturel, L. Exhaust emissions of PAHs of passenger cars. Polycyclic Aromatic Compounds, v.26, p.69-78, 2006.

26. Rojas, N. Y.; Milquez, H. A.; Sarmiento, H. Characterizing priority polycyclic aromatic hydrocarbons (PAH) in particulate matter from diesel and palm oil-based biodiesel B15 combustion. Atmospheric Environment, v.45, p.6158-6162, 2011.

27. Marr, L. C.; Kirchstetter, T. W.; Harley, R. A. Characterization of polycyclic aromatic hydrocarbons in motor vehicle fuels and exhaust emissions. Environment Science Technology, v.33, p.3091-3099, 1999.

28. Alkurdi, F.; Karabet, F.; Dimashki, M. Characterization, concentrations and emission rates of polycyclic aromatic hydrocarbons in the exhaust emissions from in-service vehicles in Damascus. Atmospheric Research, v.120-121, p.68-77, 2013.

29. Jones, C. C.; Chughtai, A. R.; Murugaverl, B.; Smith, D. M. Effects of air/fuel combustion ratio on the polycyclic aromatic hydrocarbon content of carbonaceous soots from select fuels. Carbon, v.42, p.2471-2484, 2004.

30. Brasser, L. J. Polycyclic aromatic hydrocarbon concentrations in the Netherlands. VDIBerichte, v.348, p.171-180, 1980.

31. Hooper, M. A.; Body, P. J.; Hooper, B. M. Coal Liquefaction: Atmospheric Impacts: a Final Report to the Coal Corporation of Victoria. Gippsland Centre for Environmental Science, Monash University, Gippsland, 1993.

32. Smith, D. J. T.; Harrison, R. M. Polycyclic aromatic hydrocarbons in atmospheric particles. In: Harrison, R.M., Van Grieken, R. (Eds.). Atmospheric Particles, Wiley, 1998.

33. Ravindra, K.; Bencs, L.; Wauters, E.; Hoog, J. de.; Deutsch, F.; Roekens, E.; Bleux, N.; Berghmans, P.; Grieken, R. van. Seasonal and site-specific variation in vapour and aerosol phase PAHs over Flanders (Belgium) and their relation with anthropogenic activities. Atmospheric Environment, v.40, p.771-785, 2006.

34. Miguel, A. H.; Kirchstetter, T. W.; Harley, R. A. On-Road emissions of particulate polycyclic aromatic hydrocarbons and Black Carbon from Gasoline and diesel vehicles. Environment Science Technology, v.32, p.450-455, 1998.

35. Harrison, R. M.; Smith, D. J. T.; Luhana, L. Source apportionment of atmospheric polycyclic aromatic hydrocarbons collected from an urban location in Birmingham, U.K. Environment Science Technology, v.30, p.825-832, 1996.

36. Bourotte, C.; Forti, M. C.; Taniguchi, S.; Bícego, M. C.; Lotufo, P. A. A wintertime study of PAHs in fine and coarse aerosols in São Paulo city, Brazil. Atmospheric Environment, v.39, p.37993811, 2005. 
37. Simcik, M. F.; Eisenreich, S. J.; Lioy, P. J. Source apportionment and source/sink relationships of PAHs in the coastal atmosphere of Chicago and Lake Michigan. Atmospheric Environment, v.33, p.5071-5079, 1999.

38. De Martinis, B. S.; Okamoto, R. A.; Kado, N. Y.; Gundel, L. A.; Carvalho, L. R. F. Polycyclic aromatic hydrocarbons in a bioassay-fractionated extract of PM10 collected is São Paulo, Brazil. Atmospheric Environment, v.36, p.307-314, 2002.

39. Mainwaring, S. J.; Stirling, D. M. A study of the size distribution and concentrations of polynuclear hydrocarbons in Melbourne air. In: Webb, K.A., Smith, A.J. (Eds.), Proceedings of the 7th International Clean Air Conference, Adelaide, Australia. Ann Arbor Science, Ann Arbor, MI, p.605-622, 1981.

40. Azimi, S.; Rocher, V.; Muller, M.; Moilleron, R.; Thevenot, D. R. Sources, distribution and variability of hydrocarbons and metals in atmospheric deposition in an urban area (Paris, France). Science of the Total Environment, v.337, p.223-239, 2005.

41. Manoli, E.; Vousta, D.; Samara, C. Chemical characterization and source identification/ apportionment of fine and coarse air particles in Thessaloniki, Greece. Atmospheric Environmental, v.36, p.949- 61, 2002.

42. Park, J. S.; Wade, T. L.; Sweet, S. Atmospheric distribution of polycyclic aromatic hydrocarbons and deposition to Galveston Bay, Texas, USA. Atmospheric Environment, v.35, p.3241-3249, 2001.

43. Lang, Q.; Zhang, Q.; Jaffé, R. Organic aerosols in the Miami area, USA: temporal variability of atmospheric particles and wet/dry deposition. Chemosphere, v.47, p.427- 441, 2002.

44. Khalili, N. R.; Scheff, P. A.; Holsen, T. M. PAH source fingerprints for cake ovens, diesel, and gasoline engines, highway tunnel, and wood combustion emissions. Atmospheric Environment, v.29, n.4, p.533-542, 1995.

45. Soclo, H. H.; Garrugues, P.; Ewald, M. Origin of polycyclic aromatic hydrocarbons (PAHs) in coastal marin sediments: case studies in Cotonou (Benin) and Aquitaine (France) areas. Mar Pollut Bull, v.40, p.387-396, 2000.

46. Moreda, J. M.; Arranz, A.; Fdez De Betono, S.; Cid, A.; Arranz, J. F. Chromatographic determination of aliphatic hydrocarbons and polyaromatic hydrocarbons (PAHs) in a sewage sludge. Science of the Total Environment, v.220, p.33-43, 1998.

47. Vasconcellos, P. C.; Zacarias, D.; Pires, M. A. F.; Pool, C. S.; Carvalho, L. R. F. Measurements of polycyclic aromatic hydrocarbons in airborne particles from the metropolitan area of São Paulo City, Brazil. Atmospheric Environment, v.37, p.3009-3018, 2003.

48. Fernandes, M. B.; Brickus, L. S. R.; Moreira, J. C.; Cardoso, J. N. Atmospheric BTX and polyaromatic hydrocarbons in Rio de Janeiro, Brazil. Chemosphere, v.47, p.417-425, 2002.

49. Teixeira, E. C.; Agudelo-Castañeda, D. M.; Fachel, J. M. G.; Leal, K. A.; Garcia, K. de O.; Wiegand, F. Source identification and seasonal variation of polycyclic aromatic hydrocarbons associated with atmospheric fine and coarse particles in the Metropolitan Area of Porto Alegre, RS, Brazil. Atmospheric Research, v. 118, p.390-403, 2012.

50. Ré-Poppi, N.; Silva, M. S. Polycyclic aromatic hydrocarbons and other selected organic compounds in ambient air of Campo Grande City, Brazil. Atmospheric Environment, v.39, p.2839-2850, 2005. 
51. Guo, H.; Lee, S. C.; Ho, K. F.; Wang, X. M.; Zou, S. C. Particle-associated polycyclic aromatic hydrocarbons in urban air of Hong Kong. Atmospheric Environment, v.37, p.5307-5317, 2003.

52. Liu, L-B.; Liu, Y.; Lin, J-M.; Tang, N.; Hayakawa, K.; Maeda, T. Development of analytical methods for polycyclic aromatic hydrocarbons (PAHs) in airborne particulates: A review. Journal of Environmental Sciences, v.19, p.1-11, 2007.

53. Anastasopoulos, A. T.; Wheeler, A. J.; Karman, D.; Kulka, R. H. Intraurban concentrations, spatial variability and correlation of ambient polycyclic aromatic hydrocarbons (PAH) and PM2.5. Atmospheric Environment, v.59, p.272-283, 2012.

54. Chen, Y.; Ho, K. F.; Hang Ho, S. S.; Kei Ho, W.; Lee, S. C.; Zhen Yu, J.; Sit, E. H. L. Gaseous and particulate polycyclic aromatic hydrocarbons (PAHs) emissions from commercial restaurants in Hong Kong. Journal of Environmental Monitoring, v.9, p.1402-1409, 2007.

55. Dejean, S.; Raynaud, C.; Meybeck, M.; Massa, J. P. D.; Simon, V. Polycyclic aromatic hydrocarbons (PAHs) in atmospheric urban area: monitoring on various types of sites. Environmental Monitoring and Assessment, v.148, p.27-37, 2009.

56. Menichini, E.; lacovella, N.; Monfredini, F.; Turrio-Baldassarri, L. Relationships between indoor and outdoor air pollution by carcinogenic PAHs and PCBs. Atmospheric Environment, v.41, p.9518-9529, 2007.

57. Nelson, P. F.; Tibbett, A. R.; Day, S. J. Effects of vehicle type and fuel quality on real world toxic emissions from diesel vehicles. Atmospheric Environment, v.42, p.5291-5303, 2008.

58. Park, S. S.; Kim, Y. J.; Kang, C. H. Atmospheric polycyclic aromatic hydrocarbons in Seoul, Korea. Atmospheric Environment, v.36, p.2917-2924, 2002.

59. Cincinelli A.; Del Bubba M.; Martellini, T.; Gambaro A.; Lepri, L. Gas-particle concentration and distribution of n-alkanes and polycyclic aromatic hydrocarbons in the atmosphere of Prato (Italy). Chemosphere, v.68, p.472-478, 2007.

60. Gigliotti, C. L.; Totten, L. A.; Offenberg, J. H. Atmospheric concentrations and deposition of polycyclic aromatic hydrocarbons to the mid-atlantic east coast region. Environmental Science and Technology, v.39, p.5550-5559, 2005.

61. Oda, J.; Nomura, S.; Yasuhara, A.; Shibamoto, T. Mobile sources of atmospheric polycyclic aromatic hydrocarbons in a roadway tunnel. Atmospheric Environment, v.35, p.4819-4827, 2001.

62. Borrás, E.; Tortajada-Genaro, L. A. Characterisation of polycyclic aromatic hydrocarbons in atmospheric aerosols by gas chromatography-mass spectrometry. Analytica Chimica Acta, v.583, p.266-276, 2007.

63. Godoi, A. F. L.; Ravindra, K.; Godoi, R. H. M.; Andrade, S. J.; Santiago-Silva, M., Van Vaeck, L.; Van Grieken, R. Fast Chromatographic determination of polycyclic aromatic hydrocarbons in aerosol samples from sugar cane burning. Journal of Chromatography A, v.1027, p.49-53, 2004.

64. Karthikeyan, S.; Balasubramanian, R.; See, S.W. Optimization and validation of a low temperature microwave-assisted extraction method for analysis of polycyclic aromatic hydrocarbons in airborne particulate matter., Talanta, v.69, p.79-86, 2006. 
65. Ho, K. F.; Ho, S. S. H.; Lee, S. C.; Cheng, Y.; Chow, J. C.; Watson, J. G.; Louie, P. K. K.; Tian, L. Emissions of gas- and particle-phase polycyclic aromatic hydrocarbons (PAHs) in the Shing Mun Tunnel, Hong Kong. Atmospheric Environment, v.43, p.6343-6351, 2009.

66. International Agency for Research on Cancer (IARC). Evaluations of carcinogenicity to humans. Some non-heterocyclic polycyclic aromatic hydrocarbons and some related exposures/IARC. Working Group on the Evaluation of Carcinogenic Risks to Humans (2005: Lyon, France), v.92, 2005.

67. Cerna, M.; Pochamanova, D.; Pastorkova, A.; Bene, I.; Lenicek, J.; Topinka, J.; Binkova, B. Genotoxicity of urban air pollutants in Czech Republic. Muation Research/Genetic Toxicology and Environmental Mutagenesis, v.469, n.1, p.71-82, 2000.

68. Durant, J. L.; Bushy, W. F.; Lafleur, A. L.; Penman, B. W.; Crespi, C. L. Human cell mutagenicity of oxygenated, nitrated and unsubstituted polycyclic aromatic hydrocarbons associated with urban aerosols. Mutation Research-Genetic Toxicology, v.371, n. 3-4, p.123-157, 1996.

69. Dutch National Institute of Public Health and the Environment (RIVM). Environmental risk limits in The Netherlands. Report no. 601640 001, 1999.

70. Dutch National Institute of Public Health and the Environment (RIVM). Setting integrated environmental quality standards: environmental quality standards for soil, water and air, 1999.

71. Agency for Toxic Substances and Disease Registry (ATSDR). Toxicological Profile for Polycyclic Aromatic Hydrocarbons. US Department of Health and Human Services, Public Health Service, 1995.

72. European Union Directive (EUD). Directive 2004/107/EC of the European parliament and of the council. Official Journal of European Union L23, p.3-16, 2004.

73. National Air Quality Information Archive (NAQIA). United Kingdom, 1999.

74. Paturel, L.; Saber, A.; Combet, E.; Joumard, R. Analysis of PAH emissions from passenger cars by high resolution Shpol'skii spectrofluorimetry. Polycyclic Aromatic Compounds, v.9, p.331339, 1996.

75. Schauer, J. J.; Kleeman, M. J.; Cass, G. R.; Simoneit, B. R. T. Measurement of emissions from air pollution sources. 5. C1-C32 organic compounds from gasoline-powered motor vehicles. Environment Science Technology, v.36, p.1169-1180, 2002.

76. Rogge, W. F.; Mazurek, M. A.; Hildemann, L. M.; Cass, G. R. Quantification of urban organic aerosols at a molecular level: identification, abundance and seasonal variation. Atmospheric Environment, v.27A, n.8, p.1309-1330, 1993.

77. Ying, W.; Longbao, Z.; Hewu, W. Diesel emission improvements by the use of oxygenated DME/diesel blend fuels. Atmospheric Environment, v.40, p.2313-2320, 2006.

78. Abrantes, R.; Assunção, J. V.; Pesquero, C. R.; Bruns, R. E.; Nóbrega, R. B. Emission of polycyclic aromatic hydrocarbons from gasohol and ethanol vehicles. Atmospheric Environment, v.43, p.648-654, 2009.

79. Westerholm, R.; Alsberg, T.; Frommelin, A.; Strandell, M.; Rannug, U.; Winquist, L.; Grigoriadis, V.; Egebäck, K. -E. Effect of fuel polycyclic aromatic hydrocarbon content on the emissions of polycyclic aromatic hydrocarbons and other mutagenic substances from a gasoline-fueled automobile. Environmental Science and Technology, v.22, p.925-930, 1988. 
80. He, C.; Ge, Y.; Tan, Y.; You, K.; Han, X.; Wang, J. Characteristics of polycyclicaromatic hydrocarbons emissions of diesel engine fueled with biodiesel and diesel, Fuel, v.89, ed.8, p.2040-2046, 2010.

Surawski, N. C.; Miljevic, B.; Ayoko,G. A.; Elbagir,S.; Stevanovic, S.; Fairfull-Smith, K. E.; Bottle, S. E.; Ristovski, Z. D. Physicochemical Characterization of Particulate Emissions from a Compression Ignition Engine: The Influence of Biodiesel Feedstock. Environmental Science Technology, v.45, p.10337-10343, 2011. 